\title{
Preventable readmissions and physician notification
}

\author{
Daniel Kazzaz \\ Secure Exchange Solutions, USA \\ Correspondence: Daniel Kazzaz. Address: 1700 Research Blvd., Suite 220, Rockville, MD 20850, USA. E-mail: \\ dan.kazzaz@SecureExSolutions.com
}

Received: March 13, 2013

Accepted: August 14, $2013 \quad$ Online Published: August 27, 2013

DOI : $10.5430 /$ jha.v3n1p1

URL: http://dx.doi.org/10.5430/jha.v3n1p1

\section{Abstract}

Preventable hospital readmissions burden our health system with excessive costs. The key to reducing readmissions is appropriate follow-up care for patients upon discharge. Many patients today do not receive necessary follow-up care for the simple reason that their primary care and specialist physicians are not made aware of the hospitalization. A simple and scalable solution is needed to provide automatic notifications to physicians when their patients are admitted to and discharged from hospitals. Such systems, based on national standards for universal adoption, are emerging in several forms, making headway in reducing preventable hospital readmissions.

\section{Key words}

Preventable readmissions, Care continuity, Provider notification

\section{I ntroduction}

Engage a conversation today with any group of physicians about "shared risk" or "accountable care" and you will quickly learn of frustrations centered around costly preventable hospital readmissions. In the field, you will hear stories about patients who have been admitted then discharged from hospitals without the primary care and specialty physicians being notified. A cardiologist will tell you of the patient she recently discovered who, months earlier, had visited an ER with chest pain. Frustrated patients will complain that they did not see the doctors they know and trust during a hospital stay.

A recent study ${ }^{[1]}$ has shown that the risk of readmission increases ten-fold for patients not seen by their primary care physician within 30 days of hospital discharge. In spite of the increased risk, one third of primary care physicians today never learn that their patient was hospitalized. This problem has led to an increased emphasis on outpatient follow-up to avoid hospital readmissions, highlighting the need for improved and timely communications between hospitals, physicians and patients, all of whom are seeking to improve the delivery of care.

\section{The problem - Expensive preventable readmissions}

Readmissions are expensive for all parties involved, exceedingly so, costing Medicare alone more than \$17 billion per year ${ }^{[1]}$. As a direct result, the Centers for Medicaid and Medicare Services (CMS) have instituted penalties for preventable readmissions ${ }^{[2]}$. In 2012, the first year of the Readmissions Reduction Program, which is part of the Patient Protection and Affordable Care Act, penalties alone cost 2,211 hospitals a total of \$280 million, or an average of \$12,664 per hospital ${ }^{[3]}$. 
Most stakeholders agree that improving care coordination is a key remedy for preventable readmissions. Several healthcare systems have already implemented pilots to demonstrate the benefits of improved care coordination with one essential common aspect: better hospital-to-primary care communications.

Another recent study ${ }^{[4]}$ showed that one third of primary care physicians never know their patient was hospitalized, demonstrating significant gaps in our existing communication processes. There are multiple and valid reasons why connecting primary care to the admitting hospital has been difficult:

1) A physician’s patients may be admitted to any one of multiple hospitals in a geographic area.

2) The patient may be incapacitated or have failed to communicate accurately the physician's name and contact information to the hospital staff.

3) The hospital may have neglected to seek out the information to connect with the patient’s physician.

4) The hospital may still lack the resources or infrastructure needed to communicate easily with the physician.

\section{The solution - Simple, universal communications}

To reduce preventable readmissions, hospitals and physicians require a simple, easy-to-use solution that ensures primary care physicians and specialists can be notified immediately when a patient is admitted to the hospital, discharged or transferred.

Northern Piedmont Community Care ${ }^{[5]}$, a division of North Carolina Community Care, which cares for 600 asthmatic children, implemented a program that included both provider notifications and nurse-led asthma management. After 11 months, visits to primary care physicians had increased $12 \%$, while visits to the emergency room and hospitalizations fell by $43 \%$ and $34 \%$, respectively. The total cost to Medicaid dropped by $23 \%$ during that period ${ }^{[1]}$. This example illustrates the path to reducing preventable readmissions, but does not present a solution for the universal connectivity needed to ensure that all providers, in all networks, are notified when their patients are admitted to a hospital.

The emergence of universal patient attribution services ${ }^{[6]}$, combined with provider notification systems based on the Direct Messaging protocol ${ }^{[8]}$ established by the Office of the National Coordinator (ONC) to enable open communications among all healthcare stakeholders, offers a tremendous opportunity for rapid adoption by hospitals, primary care physicians, payers and patients. As the healthcare industry searches for secure, safe and efficient ways to exchange patient information, Direct Messaging has surfaced as an affordable, universal and easy-to-use solution ${ }^{[9]}$.

Until recently, a dependable and secure method for hospitals to send electronic notifications to community physicians did not exist. This all changed when CurrentCare ${ }^{\circledR}$, Rhode Island's statewide Health Information Exchange (HIE), launched an electronic notification solution. CurrentCare's innovation relied on guidance developed by ONC and the Rhode Island Quality Institute (RIQI). CurrentCare uses a simple and secure automated system to deliver real-time clinical notifications to physicians. Maryland's state HIE, Chesapeake Regional Information System for our Patients (CRISP), now also offers automated real-time physician notifications using Direct Messaging. These notifications are a first step to timely follow-up care that helps circumvent costly hospital readmissions.

Provider Notification systems, such as those offered by CurrentCare ${ }^{[7]}$ and CRISP, can be improved with the addition of provider-patient communications. Such improvements are readily achievable using Direct Messaging and may further reduce readmissions by increasing patient accountability and offering hospitals and providers a simple tool to deliver healthcare information and guidance to patients. 
Since Provider Notification systems are mostly implemented in conjunction with HIEs, which already exist in every state, additional charges associated with the enhancement are minimal. A region may spend $\$ 500$ thousand to implement. In Maryland, for example, with 40 hospitals, we estimate the average start-up cost at approximately $\$ 12 \mathrm{~K}$ per hospital. Annual subscription fees are typically $40 \%$ of the initial cost, therefore an ongoing fee of approximately $\$ 5 \mathrm{~K}$ per year per hospital.

In addition to avoiding penalties for preventable readmissions, hospitals implementing notification systems may also receive additional incentives from CMS. As of January 1, 2013, commenced reimbursement of two new Transition of Care Management codes, CPT codes 99495 and $99496^{[8]}$.

Both codes require communication (in-person contact, telephone or electronic) with the patient and/or caregiver within two business days of discharge.

Both codes require medical decision making of moderate complexity and high complexity respectively during the service period (low complexity decisions are not part of these codes).

Both codes require a face-to-face visit within 14 calendar days and 7 calendar days respectively of discharge.

CMS Reimbursements for the codes range from \$135 to \$231 per patient.

\section{Current capabilities explained}

Communications between the participating Rhode Island hospitals and Thundermist Health Center, one of Rhode Island's largest and most innovative medical groups, illustrates Rhode Island's current provider notification process.

When a patient is admitted to a hospital, an admission message (technically known as an HL7 ADT) is sent to HIE ${ }^{[9]}$, in this case CurrentCare or CRISP. The admission message then becomes part of the patient's longitudinal record and replaces the traditional notification facsimile to primary care or specialty physicians identified by the patient. The HIE's software reformats and forwards the admission message, in a humanly readable format, directly to the patient's primary care physician using Direct Messaging.

The Figure is an example of the flow of information from hospital to physician when notifying a provider that a patient has been admitted or discharged.

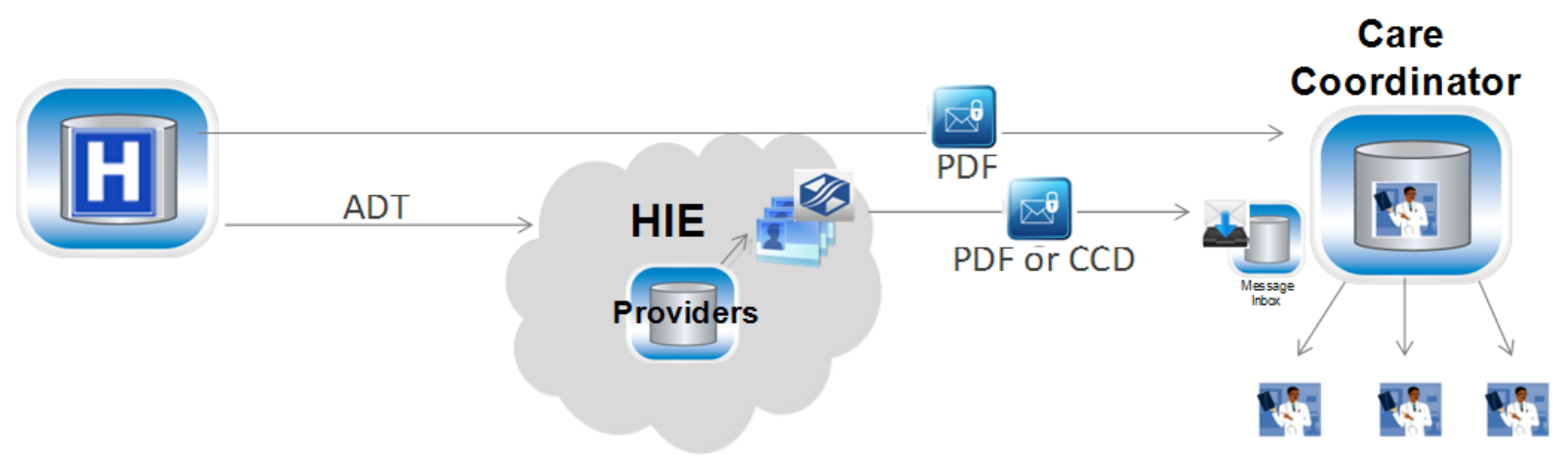

Figure. Sample physician notification system

CurrentCare notifies the primary care physician of patient admission and discharge in the following manner: 
1) Extracts the patient name, physician name, and reason for admission.

2) Locates the patient in the database to determine whether the patient has opted-in for sharing clinical information. (This step is only necessary in states, such as Rhode Island, where patients must “opt-in” to the exchange.)

3) Searches for the physician on file and determines if a secure message can be delivered.

4) Transforms the admission or discharge message into both PDF and CCD (Continuity of Care Document) formats. The CCD format is supported by all Electronic Health Records (EHRs), and many also support seamless consumption of a CCD that arrives through secure email.

5) Pushes the patient and admission information to the primary care physician, using Direct Messaging.

The CRISP notification system ${ }^{[10]}$, which is highly utilized among Maryland providers, differs from that of CurrentCare in the following ways:

1) CRISP employs a precursory step whereby the physicians and other care providers upload the list of patients for which they wish to be notified.

2) Once the patient information is extracted from the Admission/Discharge/Transfer (ADT) report, they locate the appropriate physician. Since the physician will already have a Direct-enabled account, CRISP knows they can be notified.

Both the CurrentCare and CRISP systems would benefit from the addition of an assignment mechanism whereby the patient may select the providers (also caregivers and family members) to be notified of the hospital admission, discharge or transfer.

Most HIEs allow physicians to search the database for patients. While such queries provide access to a longitudinal patient record, the process could be further augmented with a push of key information to the physician as the end point. Currently, one of the approved methods of sending secure messages to physicians is via Direct Messaging. Direct Messaging is a secure email protocol that transfers the message as a secure file transport, or makes it accessible via a secure web portal. The following three scenarios illustrate how the use of the Direct Messaging is aligned with the current needs of physicians:

\subsection{Physicians with EHRs but without a fax server in the practice}

The physician endpoint has an EHR but does not have a fax server. Facsimiles must be printed in hard copy then scanned into the EHR. The image of the document must then be attached to the patient's record and queued up for the doctor to review. The hard copy must be destroyed or retained in a paper record filing system. A Direct Messaging email or file transfer reduces the number of steps involved in the workflow, as well as reducing the required manual intervention and waste.

\subsection{Physicians with EHRs and a fax server in the practice}

The physician has an EHR record and a fax server so that each fax is received as a separate electronic image. Each image must be opened, the patient record found, the image attached to the patient's record then queued up for the doctor to review. The inherent low-resolution fax transmission may gray out or blur critical details in the image. Using Direct Messaging, faxed documents and PDFs arrive in the same directory, leveraging the existing workflow for processing incoming documents. When a patient name is embedded within the PDF file name in an email, the process is further optimized by eliminating the need to manually review the file to locate the patient name. 


\subsection{Physicians with EHRs fully implemented}

When the EHR system can import and consume CCDs, the CCD file arrives at the server via Direct Messaging and the data is consumed by the EHR. The file is attached to the patient record automatically and queued for the doctor to review. Direct compliance enables universal communications across different EHRs, eliminating costly proprietary interfaces.

\section{Physician-Patient communication}

The current HIE-to-physician notification process relies on:

1) Patient consent at the HIE (applicable only in states that require an opt-in);

2) Accurate patient and doctor information found within the Admission/Discharge/Transfer message;

3) The physician’s ability to receive provider notifications via Direct Messaging.

It is now possible to streamline these three items by applying a patient attribution service ${ }^{[11]}$. Based on social networking infrastructures, patients register in a self-service model or through their physician's office via the Internet. Patients can indicate physicians and caregivers in their network, as well as family members, who should be notified in case of an emergency. Linked with the existing HIE patient repository, this information provides the HIE with a physician-patient linkage to fill gaps in the current ADT message. This allows, for example, a cardiac patient's arrival at an emergency room to trigger an immediate notification to both his primary care physician and cardiologist.

Creating a communications link between providers and patients also contributes to patient engagement and gives physicians a way to send patients instructions for follow-up care.

\section{Conclusion}

Improved care coordination can be achieved through effective communications between hospitals and physicians. A recent article in the Southern Medical Journal ${ }^{[1]}$ noted that in one-third of hospitalizations, primary care physicians were not notified that their patients had been hospitalized. Another study ${ }^{[1]}$ showed readmissions increasing ten-fold for patients not seen by their physician within 30 days of discharge. As hospital-based medicine becomes more prevalent, communications between physicians are becoming increasingly unreliable, and current strategies in place to prevent readmissions, such as the use of case managers and social workers, are not delivering the impact achievable through the use of Direct Messaging communications.

The use of secure electronic provider notification systems can be a major contributor to reducing preventable hospital readmissions, offering significant cost savings to payers as the cost of implementing notification systems, enhanced with next-generation patient attribution services, dwarfs even the annual average the cost of readmission penalties, not to mention the costs to CMS of preventable readmission stays.

\section{References}

[1] William P. Moran, MD, MS, Kimberly S. Davis, MD, Thomas J. Moran, MSW, Roger Newman, MD, Patrick D. Mauldin, PhD. Where Are My Patients? It is Time to Automate Notification of Hospital Use to Primary Care Practices. Southern Medical Journal. [Internet] 105: 1 (2012): 18-23. Available from: http://www.ncbi.nlm.nih.gov/pubmed/22189662 or http://www.medscape.com/viewarticle/756372_print. 17 July 2013

[2] Pittman, David. All Readmissions May Trigger \$\$ Penalties. [Internet] May 17, 2013. Available from: http://www.medpagetoday.com/HospitalBasedMedicine/GeneralHospitalPractice/39226

[3] Herman, Bob. High Readmissions Cost More Than 2k Hospitals \$280M in Medicare Funds. Becker’s Hospital Review. [Internet] 14 August 2012. Available from:

Published by Sciedu Press 
http://www.beckershospitalreview.com/racs-/-icd-9-/-icd-10/high-readmissions-cost-more-than-2k-hospitals-280m-in-medicare-f unds.html

[4] Arora VM, Prochaska ML, Farnan JM, et al. Problems after discharge and understanding of communication with their primary care physicians among hospitalized seniors: a mixed methods study. J Hosp Med. [Internet] 5:7 (2010): 385-391. Available from: http://www.ncbi.nlm.nih.gov/pubmed/20578045. 17 August 2013

[5] Northwest Piedmont, CCNC. [Internet] 1 August 2013. Northern Piedmont Community Care is a network of five hospitals and 41 primary care practices covering six counties with the larger network of Community Care of North Carolina. Available from: http://www.communitycarenc.com/our-networks/\#npiedmont

[6] Physician and Other Medical Provider Grouping and Patient Attribution Methodologies. [Internet] August 2013. Available from: http://www.cms.gov/Medicare/Quality-Initiatives-Patient-Assessment-Instruments/GEM/downloads/GEMMethodologies.pdf. 17

[7] Current Care. Thundermist Health Video. Online Video. [Internet] 17 August, 2013. Available from: www.thundermisthealth.org/NewsEvents/ViewArticle/tabid/164/smid/605/ArticleID/7/reftab/162/t/Information-About-CurrentC are/Default.aspx

[8] American Academy of Family Physicians, AAFP. Final 2013 Medicare Physician Fee Schedule. [Internet] 2012; 7. Available from: http://www.aafp.org/online/etc/medialib/aafp_org/documents/policy/fed/background/2013feeschedule.Par.0001.File.tmp/Final20 13MPFS110812.pdf

[9] Health Information Exchanges and the State Health Information Exchange Cooperative Agreement Program. [Internet] 17 August 2013. Available from: http://www.healthit.gov/policy-researchers-implementers/state-health-information-exchange

[10] CRISP: ENS - Encounter Notification System. [Internet] 17 August 2013. Available from: http://www.crisphealth.org/LinkClick.aspx?fileticket=PPKbVlpNstQ\%3D\&tabid=272

[11] ONC Health IT Dashboard. [Internet] 17 August, 2013. Available from: http://dashboard.healthit.gov/ 